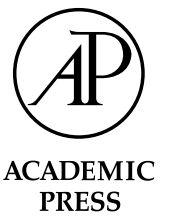

\title{
Can implementation intentions help to overcome ego-depletion?
}

\author{
Thomas L. Webb* and Paschal Sheeran \\ Department of Psychology, University of Sheffield, Sheffield S10 2TN, UK
}

Received 4 September 2001; revised 14 May 2002

\begin{abstract}
Research on ego-depletion suggests that the ability to self-regulate one's behavior is limited: Exerting self-control on an initial task reduces performance on a subsequent task that also requires self-control. Two experiments tested whether forming implementation intentions could prevent ego-depletion and/or offset the effects of ego-depletion. Experiment 1 found that participants who formed implementation intentions during an initial ego-depleting task subsequently showed greater persistence on an unsolvable puzzles task compared to participants who did not form implementation intentions. Experiment 2 found that among participants who had been ego-depleted during an initial task, forming implementation intentions improved subsequent performance on a Stroop task to the level exhibited by non-depleted controls. Thus, implementation intentions help to enhance people's ability to self-regulate their behavior.
\end{abstract}

(c) 2003 Elsevier Science (USA). All rights reserved.

Keywords: Ego-depletion; Implementation intentions; Self-regulation; Stroop task

The ability to regulate impulses, emotions, and desires is one of the core features of the self (Tice, Bratlavsky, \& Baumeister, 2001). Exerting self-control allows us to behave flexibly, to override undesirable responses, and to avoid temptation. In a review of the literature, Baumeister, Heatherton, and Tice (1994) concluded that failure to self-regulate plays a central part in the majority of personal (e.g., heroin addiction) and social (e.g., crime) problems in Western societies. Muraven and Baumeister (2000) proposed that selfregulatory failure occurs because self-control is a limited resource akin to strength or a muscle. If this limited resource is depleted then the person can no longer regulate her behavior effectively. Hence, preventing or offsetting such depletion could greatly benefit people.

"Ego-depletion" is the term used to describe the temporary depletion of self-regulatory capacity by an initial act of self-control. Baumeister, Bratlavsky, Muraven, and Tice (1998) employed a number of ego-depletion paradigms to demonstrate the limits on people's self-regulatory capacity. For example, Experiment 1 showed that being told to eat radishes, rather than

\footnotetext{
${ }^{*}$ Corresponding author.

E-mail address: pcp00tlw@shef.ac.uk (T.L. Webb).
}

available chocolate, reduced participants' subsequent persistence with unsolvable puzzles. Similarly, Muraven, Tice, and Baumeister (1998) observed that thought suppression (e.g., about white bears) reduced participants' subsequent ability to suppress their laughter when watching a funny video, and, in a separate experiment, their persistence on unsolvable anagrams. It appears that exercising control in one domain depletes one's capacity to exercise control in a second domain.

The self-control strength model (Muraven \& Baumeister, 2000) proposes that exerting self-control consumes self-control strength and thereby reduces the amount of strength that is available for subsequent selfcontrol. Moreover, it appears that cognitive, emotional, and physical acts of self-control all draw upon the same limited resource (Muraven \& Baumeister, 2000). It is important to note that not all effortful behaviors involve self-control. For example, solving maths problems involves considerable cognitive effort, but does not require overriding a response, and hence does not result in egodepletion (Muraven et al., 1998).

Although the exact nature of the self-control resource is unclear, it is linked to physical tiredness (Baumeister et al., 1998). For example, in all four experiments reported by Muraven et al. (1998), ego-depleted participants 
reported increased fatigue compared to their nondepleted counterparts. However, Baumeister et al. (1998) noted that diminished capacity for self-control is not the same thing as physical fatigue-fatigue is only a marker for the strength of the self-control resource. Moreover, unlike fatigue, alternative explanations of ego-depletion such as mood or learned helplessness have not been empirically supported (Muraven \& Baumeister, 2000).

The present research examines how ego-depletion might be prevented, and, furthermore, how the effects of ego-depletion might be offset once they have occurred. We propose that "implementation intentions" may provide a strategy that is helpful in overcoming egodepletion.

Implementation intentions (Gollwitzer, 1993, 1996, 1999) are sub-ordinate to goal intentions and refer to statements of the form: "As soon as situation $y$ occurs, I will initiate goal-directed behavior $x$." By specifying when, where, and how one will act, implementation intentions pass control of behavior to anticipated environmental cues. Passing control to specified cues means that the need for cognitive control is circumvented-a process referred to as "strategic automatization" (Gollwitzer \& Schaal, 1998, p. 124).

Evidence suggests that implementation intentions are effective in promoting goal achievement. A meta-analysis of 15 studies by Sheeran (2002) found that implementation intentions had a "medium" effect size on behavior $(r=.33)$. Moreover, effectiveness has been demonstrated for a range of behaviors, from infrequently performed behaviors such as attendance for cervical cancer screening (Sheeran \& Orbell, 2000) to repeated behaviors that are performed daily such as vitamin supplement use (Sheeran \& Orbell, 1999), and across a range of samples and measures of behavior (Sheeran, 2002).

Why are implementation intentions effective? According to Gollwitzer (1999), forming an implementation intention means that as soon as the environmental cue specified in the implementation intention is encountered, the intended behavior will be initiated automatically. Gollwitzer and Schaal (1998) argue that the mental representation of the anticipated situational cue becomes highly accessible when implementation intentions are formed, and that this has a number of consequences. First, this heightened accessibility serves to improve detection of the situational cues (Stellar, 1992, cited in Gollwitzer \& Schaal, 1998). Second, heightened accessibility serves to focus attention. For example, Gollwitzer et al. (1992, cited in Gollwitzer, 1993) used a dichotic listening paradigm to investigate how cues specified in implementation intentions attracted attention. Results showed that critical words related to anticipated opportunities to act attracted more attention than non-critical words. Moreover, subsequent recognition performance was also better for these critical words. Aarts, Dijksterhuis, and Midden (1999) showed that specifying implementation intentions to collect a coupon on the way to a cafeteria increased both the accessibility of cues related to the coupon's location (e.g., swing doors), and the likelihood of collecting a coupon. Importantly, this heightened accessibility mediated the effect of implementation intentions on performance of the behavior. It seems that implementation intentions strengthen the association between situation and behavior in memory.

Research has suggested that forming implementation intentions can inhibit the activation of automatic responses. An example is stereotypical beliefs and prejudicial feelings (Gollwitzer, Schaal, Moskowitz, Hammelback, \& Wasel, 1999, cited in Gollwitzer, 1999). Stereotypical gender attributes of females were primed using pictures of two women (named 'Ina' and 'Bea'). Some participants were asked to judge both women in a fair and non-stereotypical manner (goal intention). A subset of participants were also asked to tell themselves "whenever I see Ina, I will ignore her gender" (implementation intention). Latency of Stroop responses to gender stereotypic words served as the dependent variable. Results showed that, without implementation intentions, being primed by a picture of a woman delayed responses to stereotypical words, i.e., the gender stereotype was activated. However, forming implementation intentions suppressed the stereotypical response, and the decrement in response times for stereotypical words was not observed. Thus, implementation intentions can overcome automatic, unwanted, responses (see also Schaal, 1993, cited in Gollwitzer \& Schaal, 1998).

These findings suggest that forming implementation intentions with respect to ego-depleting tasks has the potential to offset the need for conscious control, and could, therefore, prevent or offset ego-depletion. To test this idea, the present research adopted the Stroop test (Stroop, 1935; see MacLeod, 1991, for a review) as the ego-depleting task and as the dependent variable. In the typical Stroop task, participants are presented with color words printed in ink colors that are incongruous with their meaning (e.g., the word 'green' is printed in blue ink). The participants' task is to name the ink color of each word as quickly as possible. Stroop (1935) demonstrated that response times for this task are considerably slower than for a control condition employing colored patches instead of words, indicating marked interference from incongruous color words in naming colors. Cattell $(1886$, p. 65) stated " $\ldots$ in the case of words and letters, the association between the idea and the name has taken place so often that the process has become automatic, whereas in the case of colors and pictures we must make a voluntary effort to choose the name." Thus, it seems likely that this intense voluntary effort would deplete the self-regulatory resource. However, Stroop (1935) demonstrated no interference effect from incongruously colored inks in reading the words 
themselves. Thus, unlike naming the ink color, reading the words should preserve the self-regulatory resource.

The Stroop task provides a useful method to determine if implementation intentions can overcome egodepletion for two reasons. First, successful performance of the Stroop requires the individual to interrupt an automatic habitual response (Logan, 1980), and evidence suggests that implementation intentions can be effective in this task (Gollwitzer, 1999). Second, associating a word with naming its ink color through the formation of an implementation intention should serve to heighten the accessibility of the ink color, should attract and focus attention, and thereby increase performance (Gollwitzer \& Schaal, 1998). In sum, the formation of implementation intentions to automatize Stroop performance could overcome ego-depletion.

Two experiments tested this idea. In the first experiment, implementation intentions are used to try to prevent ego-depletion. In the second experiment, implementation intentions are used to try to offset the performance decrements usually observed in egodepleted participants.

\section{Experiment 1}

\section{Method}

\section{Participants}

Fifty-one undergraduates recruited from introductory psychology courses participated in return for course credits. Participants were tested individually in a single session.

\section{Procedure}

Participants were told that the experiment investigated factors influencing people's problem-solving performance and were randomly allocated to one of three conditions, which differed only in the way that the Stroop task was presented. In the ego-depletion condition $(n=16)$, participants were asked to name the ink color of each word as quickly as possible. Participants, in the implementation intention condition $(n=16)$, were also asked to name the ink color of each word. However, in addition these participants were asked to form an implementation intention. Participants were asked to tell themselves: "As soon as I see the word I will ignore its meaning (for example, by concentrating on the second letter only) and I will name the color ink it is printed in." Participants in the control condition $(n=15)$ were simply asked to read the words.

The Stroop task comprised two A4 sheets of 154 color words (red, green, blue, and yellow), printed in 26point, uppercase, colored ink incompatible with the words' meaning. Participants in all conditions read the sheets for $10 \mathrm{~min}$.
The experimenter then informed participants that the first experiment was over, but that two experiments were being conducted in one session due to difficulties with participant recruitment. Next, in what participants believed was an ostensibly unrelated task (investigating the effects of noise on task performance), participants were given three unsolvable tracing puzzles. The puzzles (adapted from Baumeister et al., 1998) required the participant to trace the lines of a geometric shape, without retracing any lines, or removing the pen from the piece of paper. One (solvable) puzzle was demonstrated to participants before providing the test figures with the following instructions: "You can take as much time and as many trials as you want. You will not be judged on the number of trials or the time you take. You will be judged on whether or not you finish tracing the figures. If you wish to stop before you finish (i.e., solve all the puzzles), knock on the table." The participants were instructed to wear a set of headphones through which loud experimental music (Pere Ubu, 1998) on a 15-s loop was being played. This procedure served to make the task even more demanding of the self-control resource. The experimenter then left the room and-using a hidden videocamera-timed how long each participant worked on the task before giving up. Following Baumeister et al. (1998), 30 min was set as the maximum time, and participants still working at that point were stopped by the experimenter.

Participants completed the Brief Mood Introspection Scale (BMIS; Mayer \& Gaschke, 1988) and the Multidimensional Fatigue Inventory (MFI-20; Smets, A, Garssen, Bonke, \& De Haes, 1995) both after the initial Stroop task, and at the end of the experiment. The BMIS is a 16-item mood adjective scale that asks participants to indicate how well each adjective describes their current mood on a 4-point scale (definitely do not feel-definitely feel). The BMIS produces scores on two dimensions of mood: arousal and valence.

The MFI-20 is a 20 -item scale that covers five dimensions of fatigue: general fatigue, physical fatigue, mental fatigue, reduced motivation, and reduced activity. The participant is asked to indicate on a 5-point scale the extent to which they agree with the statements (yes, that is true-no, that is not true). The instructions for the fatigue scale were modified to ensure that participants reported how they felt at that particular moment by replacing "have been feeling lately" with "feel right now." The MFI-20 has been found to have good internal consistency and validity (Smets et al., 1995).

At the end of the experiment, participants completed a short manipulation check questionnaire to assess their perceptions of the tasks. Four items measured on 7point scales were presented: "How difficult did you find performing the color naming task:?" (very easy-very difficult), "How much effort did it take to perform the color naming task?" (very little effort-a great deal of 
effort), "How strong a desire did you feel to stop working on the puzzle task?" (not very strong-very strong), and "How much did you feel you were forcing yourself to work on the puzzle task?" (not at all-a great deal). Finally, participants were debriefed using a modification of the funnel debriefing procedure (Chartrand \& Bargh, 1996). None of the participants reported awareness that the two tasks were related.

\section{Results}

\section{Manipulation checks}

Analysis of variance (ANOVA) showed a significant effect of condition on the perceived difficulty of the Stroop task, $F(2,44)=33.74, p<.001$. Pairwise comparisons indicated that participants in the control condition found the task significantly easier $(M=-1.13)$ than those in the ego-depletion condition $(M=1.69)$, and implementation intention condition $(M=1.94)$. There was no difference between ego-depletion and implementation intention conditions. ANOVA also showed a significant effect of condition on the effort item, $F(2,44)=27.86, p<.001$. Participants in the control condition reported that the Stroop task required significantly less effort $(M=-0.47)$ than participants in both the ego-depletion $(M=1.88)$ and implementation intention conditions $(M=2.25)$ whereas there was no difference between participants in the ego-depletion and implementation intention conditions.

\section{Persistence}

The dependent measure was the length of time participants spent on the unsolvable puzzles (see Table 1). A one-way ANOVA indicated significant variation among the three conditions, $F(2,44)=5.12, p<.01$. Post hoc tests revealed that the ego-depletion group $(M=16.55)$ persisted for significantly less time compared to both the control group $(M=23.77)$ and the implementation intentions group $(M=23.11)$ whereas the control and the implementation intention groups did not differ in their persistence.

\section{Desire to quit}

Participants were asked two questions about the puzzle task. "How strong a desire did you feel to stop

Table 1

Persistence with unsolvable puzzles by condition: Experiment 1

\begin{tabular}{lll}
\hline Condition & Time $(\mathrm{min})$ & $S D$ \\
\hline Ego-depletion & 16.55 & 8.96 \\
Implementation intention & 23.11 & 5.76 \\
Control & 23.77 & 5.74 \\
\hline
\end{tabular}

working on the puzzle task?" and "How much did you feel you were forcing yourself to work on the puzzle task?" No significant differences between conditions were found on either of these items, $F \mathrm{~s}(2,44)=0.61$ and 1.32 , respectively, $n s$.

\section{Fatigue}

A 3 (condition: implementation intention, ego-depletion, control) $\times 2$ (time: after the Stroop task, after the puzzle task) Multivariate Analysis of Variance (MANOVA) was conducted on the five fatigue subscales of the MFI-20. There was a marginal multivariate effect for condition, $F(5,76)=1.88, p<.07$, but none of the univariate tests were significant. The multivariate test for time was significant, $F(5,38)=4.53, p<.01$. However, univariate tests showed that time only affected the reduced motivation subscale, $F(1,44)=15.88, p<.001$. Motivation was lower after the second task $(M=10.28)$ than after the first task $(M=9.26)$. The condition by time multivariate test was significant, $F(5,76)=2.02$, $p<.05$. Examination of the univariate $\mathrm{F}$ tests showed that there was one significant effect-on physical fatigue, $F(2,43)=3.99, p<.05$. Analysis of simple main effects showed that the effect of condition at time 1 (after the Stroop task) approached significance, $F(2,42)=2.96$, $p<.07$. Participants in the ego-depletion group were more physically tired $(M=13.31)$ than participants in the control group $(M=11.27)$ and the implementation intention group $(M=10.67)$. None of the other simple effects were reliable.

\section{Mood}

A 3 (condition: implementation intention, ego-depletion, control) $\times 2$ (time: after the Stroop task, after the puzzle task) MANOVA was conducted on the two mood subscales of the BMIS. There was a significant effect of time, $F(2,43)=16.77, p<.001$. Participants in all conditions were more aroused $(M=6.17)$ and felt more negative $(M=16.30)$ after the puzzle task than after the Stroop task (arousal $M=2.53$, valence $M=15.60$ ). The main effect of condition on mood, $F(4,86)=1.59$, $n s$, and the interaction between condition and time were not significant, $F(4,86)=1.76, n s$, which suggests that the differences in persistence by condition were not due to differential moods engendered by the manipulations. However, just to be sure, we reanalyzed the persistence data using mood valence and mood arousal as covariates. Findings showed that the main effect of condition remained significant when valence was covaried, $F(2,43)=5.04, p<.01$; ego-depleted participants persisted for less time $(M=16.51)$ compared to both the implementation intention $(M=23.19)$ and control $(M=23.73)$ groups, whereas the latter two groups did not differ in their persistence. Findings were identical 
when mood arousal was covaried in the analysis, $F(2,43)=5.05, p<.01(M \mathrm{~s}=16.51,23.22$, and 23.22 for the ego-depletion, implementation intention, and control conditions, respectively). In sum, the performance differences cannot be attributed to mood.

\section{Discussion}

Our first experiment obtained evidence of ego-depletion using a different combination of tasks than have been employed before. Consistent with previous findings (Baumeister et al., 1998; Muraven et al., 1998), participants who completed a Stroop task that required voluntary effort gave up sooner on a subsequent unsolvable puzzle task compared to participants who did not have to exert control on the Stroop. Importantly, however, participants who formed implementation intentions in relation to the Stroop task showed comparable persistence to control participants. It appears that implementation intentions prevented self-regulatory depletion.

Baumeister et al. (1998) noted that an alternative interpretation of this pattern of findings might be that it is adaptive to give up early on unsolvable puzzles; thus, early termination could in fact represent a higher form of self-regulation. However, this interpretation relies on the assumption that participants recognize that the puzzles were are unsolvable. Contrary to this idea, all of our participants, during the debriefing, indicated that they thought they could solve the puzzles.

Although participants' mood declined over the course of the experiment, findings showed that differences in persistence could not be attributed to mood. Instead, findings supported the idea that the state of the selfcontrol resource (and fatigue is one marker for this resource) was responsible. Comparisons indicated that ego-depleted participants were more physically tired than were participants in the control condition and participants who formed implementation intentions.

Our first experiment demonstrated that the formation of implementation intentions can prevent ego-depletion. However, what can be done to help the person who is already depleted? Experiment 2 examines whether the formation of implementation intentions can offset the decrement in subsequent performance associated with having been ego-depleted during an initial task.

\section{Experiment 2}

\section{Method}

\section{Participants}

Fifty-seven undergraduate students participated on a voluntary basis. Participants were individually tested in a single session.

\section{Procedure}

The experiment had a 2 (ego-depletion: yes, no) by 2 (implementation intentions: formed, not formed) design. Participants were randomly assigned to conditions.

In the initial task, participants in the ego-depletion condition were asked, "I would like you to do a test of mathematical ability. Please could you stand on your weaker leg and count down in sevens from 1000. It is very important to be numerically accurate." Participants in the control condition were asked, "I would like you to do a test of mathematical ability. Please could you stand normally and count to 1000 in multiples of five. It is very important to be numerically accurate." These tasks were adapted from a test used to measure automatization deficits in dyslexia (Fawcett, Nicolson, \& Dean, 1996). The task employed in the ego-depletion condition is likely to require greater self-control than the control task because of the difficulty of co-ordinating balance and a complex numerical task.

Immediately after completion of the first task, participants were asked to complete the Stroop test. The test was presented in exactly the same format as Experiment 1, except that five A4 sheets of color words were used (instead of two). The manipulation of implementation intentions was identical to that employed in Experiment 1. The time taken to complete all five cards and the error rate were recorded and served as the dependent variables.

Following completion of the second task participants were asked to fill out the Brief Mood Inventory (BMIS; Mayer \& Gaschke, 1988) and two manipulation check items; "How difficult did you find the maths task?" (7-point scale; very easy-very difficult), and "How tired did you feel after completing the color naming task?" (4-point scale; definitely did not feel-definitely felt).

Finally, participants were debriefed using a modification of the funnel debriefing procedure (Chartrand \& Bargh, 1996). None of the participants suspected performance on one task might have affected performance on the other.

\section{Results}

Manipulation check

ANOVA revealed a significant main effect of egodepletion on the perceived difficulty of the initial task, $F(1,55)=46.75, p<.001$. The ego-depletion groups $(M=2.15)$ found the initial task more difficult than did the control groups $(M=-0.17)$.

\section{Performance on the dependent variables}

The main dependent variable was how long participants took to complete the Stroop task (see Table 2). 
Table 2

Time taken to complete the Stroop and error rate by condition: Experiment 2

\begin{tabular}{|c|c|c|c|c|}
\hline \multirow{3}{*}{$\begin{array}{l}\text { Implementation } \\
\text { intention }\end{array}$} & \multicolumn{4}{|c|}{ Ego-depletion } \\
\hline & \multicolumn{2}{|l|}{ Yes } & \multicolumn{2}{|l|}{ No } \\
\hline & Mean & $S D$ & Mean & $S D$ \\
\hline \multicolumn{5}{|l|}{ Completion time } \\
\hline Formed & 11.62 & 0.93 & 11.12 & 1.08 \\
\hline Not formed & 13.91 & 1.23 & 10.88 & 1.29 \\
\hline \multicolumn{5}{|l|}{ Error rate } \\
\hline Formed & 17.15 & 5.05 & 15.38 & 5.28 \\
\hline Not formed & 27.57 & 14.71 & 15.71 & 5.48 \\
\hline
\end{tabular}

A $2 \times 2$ ANOVA revealed a significant main effect for ego-depletion, $F(1,53)=33.43, p<.001$; participants who had been depleted took significantly longer to complete the Stroop $(M=12.81)$ than participants who had not been depleted $(M=11.01)$. There was also a significant main effect for implementation intentions, $F(1,53)=9.99, p<.01$. Participants who formed implementation intentions completed the task quicker $(M=11.35)$ than participants who had not $(M=12.39)$. Finally, there was a significant interaction between ego-depletion and implementation intentions, $F(1,53)=17.32, p<.001$. Analysis of simple main effects revealed that implementation intentions affected performance for participants in the ego-depletion condition, $F(1,25)=29.31, p<.001$, but not in the control condition, $F(1,28)=0.31$, ns. Ego-depleted participants who formed implementation intentions performed significantly better than similarly depleted participants who did not form implementation intentions. We also tested the effect of ego-depletion within implementation intention conditions. Findings showed that ego-depletion affected Stroop performance when participants had not formed implementation intentions, $F(1,26)=40.55$, $p<.001$, but did not affect performance when participants had formed implementation intentions, $F(1,27)=1.78$, ns. $^{1}$

The second dependent variable was the error rate on the Stroop task. A $2 \times 2$ ANOVA revealed a significant main effect for ego-depletion, $F(1,53)=8.74, p<.01$; participants who had been depleted made more errors

\footnotetext{
${ }^{1}$ An alternative possibility is that the dual balance-and-maths task had simply induced fatigue rather than manipulated ego-depletion. To rule out this interpretation, we designed an additional condition where $n=10$ participants simply stood on their weaker leg for the same length of time as participants in the ego-depletion condition ( $M$ was $8.77 \mathrm{~min}$ ). We anticipated that participants in this condition would be fatigued, but would not be ego-depleted (since they did not have to override their tiredness and simultaneously perform the maths task). Findings were consistent with this interpretation: Participants who were fatigued (but not depleted) performed better than ego-depleted participants $(M \mathrm{~s}=11.75$ and 13.91 , respectively, $p<.01)$, and performed similarly to non-depleted participants $(M=10.88), p>.20$.
}

$(M=22.56)$ than participants who had not been depleted $(M=15.53)$. There was also a significant main effect for implementation intentions, $F(1,53)=5.03$, $p<.05$. Participants who formed implementation intentions made fewer errors $(M=16.17)$ than participants who had not $(M=21.64)$. Finally, there was a significant interaction between ego-depletion and implementation intentions, $F(1,53)=4.86, p<.05$. Findings again showed that implementation intentions affected performance for participants in the ego-depletion condition, $F(1,25)=5.87, p<.05$, but not in the control condition, $F(1,28)=.03$, ns. Ego-depleted participants who formed implementation intentions made significantly fewer errors than similarly depleted participants who did not form implementation intentions (see Table 2). Similarly, when we tested the effect of egodepletion within implementation intention conditions, findings showed that ego-depletion affected the error rate among participants who had not formed implementation intentions, $F(1,26)=7.99, p<.01$, but did not affect performance when participants had formed implementation intentions, $F(1,27)=0.85, n s$. In sum, findings for both completion time and error rate support the idea that implementation intentions can offset the effects of ego-depletion on performance.

\section{Fatigue}

Participants in the ego-depletion groups were significantly more tired after completing the Stroop task, $F(1,53)=8.59, p<.01, M=2.96$, than were participants in the non-depleted groups $(M=2.37)$. The main effect of implementation intentions and the interaction term were non-significant, $F \mathrm{~s}(1,53)=0.31$ and 0.04 , respectively, $n s$.

\section{Mood}

A MANOVA (and subsequent univariate $F$ s) on the two mood subscales of the BMIS (valence and arousal) showed a significant effect of ego-depletion on valence, $F(1,53)=4.28, p<.05$. Participants in the ego-depletion condition were in a more negative $\operatorname{mood}(M=2.93)$ than were participants in the non-depleted group $(M=5.83)$. There was no effect of implementation intentions, $F(1,53)=1.48, n s$, and the interaction between ego-depletion and implementation intentions was non-significant, $F(1,56)=0.00, n s$. The main effects and interaction term were non-significant in the case of arousal $(F \mathrm{~s}<1)$.

To ensure that the effect of ego-depletion on completion time cannot be attributed to mood, analysis of covariance (ANCOVA) was conducted using valence as a covariate. The results were unchanged. There was a significant main effect for ego-depletion, $F(1,52)=$ $35.67, p<.001$; participants who had been depleted took significantly longer to complete the Stroop $(M=$ 12.82) than participants who had not been depleted 
$(M=10.94)$ even after controlling for valence. Thus, mood does not provide an alternative explanation for the effects of ego-depletion observed here.

\section{General discussion}

This is the first demonstration of the efficacy of forming implementation intentions in overcoming egodepletion. In Experiment 1, participants first completed a Stroop task and then an unsolvable puzzle task. Participants who were ego-depleted during the Stroop task gave up quicker on the unsolvable puzzles than nondepleted participants. However, forming an implementation intention that specified a strategy for responding to incongruent Stroop stimuli increased persistence on the unsolvable puzzles to the level shown by non-depleted participants. In Experiment 2, participants first performed a balance-and-maths dual task under egodepletion versus control conditions and subsequently completed a Stroop task. Again, ego-depleted participants performed worse on the subsequent task. However, ego-depleted participants who formed implementation intentions in relation to the Stroop task performed as well as non-depleted controls. Taken together, these experiments show that forming implementation intentions can both prevent, and offset, the performance deficits associated with ego-depletion.

These findings support the strength model of selfcontrol (Muraven \& Baumeister, 2000) which argues that self-control is a limited resource. Our findings also provided support for the mechanisms that are thought to be responsible for the effects of ego-depletion. Baumeister et al. (1998) suggested that while the exact nature of the self-control resource is unknown, physical fatigue is a marker for resource depletion. Findings from both experiments were consistent with this idea; participants who had been ego-depleted were more fatigued compared to non-depleted controls. In contrast, negative mood was not reliably associated with, or a marker for, ego-depletion.

The most important finding of the present research is that forming implementation intentions can overcome the performance decrements associated with ego-depletion. In the present experiments, forming an implementation intention circumvented the need for self-control; as soon as the participant saw the word, he/she automatically named the ink color. Thus, the formation of an implementation intention removed the drain on the self-control resource usually imposed by the Stroop task. However, an important question remains, namely, why did implementation intentions only improve performance for ego-depleted participants (but not for non-depleted participants) in Experiment 2? The most plausible explanation in our view is that non-depleted participants possessed sufficient motivation to perform well on the Stroop task; forming an implementation intention conferred no extra benefit on performance for this group. In contrast, ego-depleted participants were likely to have reduced motivation so the automization of performance associated with implementation intention formation greatly enhanced these participants' ability to complete the Stroop task. Consistent with this idea, evidence suggests that implementation intentions are particularly beneficial for participants with low motivation (Brandstätter, Lengfelder, \& Gollwitzer, 2001; Schaal and Gollwitzer, 1997, cited in Gollwitzer \& Schaal, 1998), or participants who are unable to consciously deliberate (Lengfelder \& Gollwitzer, 2001).

A final issue that should be addressed concerns the implications of the present findings for research on preventing ego-depletion. Muraven, Baumeister, and Tice (1999) drew an analogy between ego-depletion and loss of muscular strength and suggested that one way to prevent ego-depletion would be to repeatedly exercise self-control, thereby training the self-control resource in a manner akin to muscle-building. In a longitudinal study, participants were asked to perform various selfcontrol exercises over a two-week period (e.g., improving posture). Compared to a no exercise control group, participants who performed the exercises showed less vulnerability to ego-depletion, as measured by performance on a handgrip task. Although self-control training is clearly effective, this self-regulatory strategy is both time-consuming and costly to the person in terms of ego-depletion during training. In contrast, the present studies have demonstrated that a single act of will can have the same benefits for the person. Moreover, implementation intentions provide a self-regulatory strategy for the participant who is already egodepleted-unlike the "muscle-building" strategy of Muraven et al. (1999).

\section{Acknowledgments}

The authors gratefully acknowledge the assistance of Luke Stableford with data collection. This research was supported by an ESRC award to the first author.

\section{References}

Aarts, H., Dijksterhuis, A., \& Midden, C. (1999). To plan or not to plan? Goal achievement or interrupting the performance of mundane behaviors. European Journal of Social Psychology, 29, 971-979.

Baumeister, R. F., Bratlavsky, E., Muraven, M., \& Tice, D. M. (1998). Ego-depletion: Is the active self a limited resource? Journal of Personality and Social Psychology, 74, 1252-1265.

Baumeister, R. F., Heatherton, T. F., \& Tice, D. M. (1994). Losing control: How and why people fail at self-regulation. London: Academic Press. 
Brandstätter, V., Lengfelder, A., \& Gollwitzer, P. M. (2001). Implementation intentions and efficient action initiation. Journal of Personality and Social Psychology, 81, 946-960.

Cattell, J. M. (1886). The time it takes to see and name objects. Mind, $11,63-65$.

Chartrand, T. L., \& Bargh, J. A. (1996). Automatic activation of impression formation and memorization goals: Non-conscious goal priming reproduces effects of explicit task instructions. Journal of Personality and Social Psychology, 71, 464-478.

Fawcett, A. J., Nicolson, R. I., \& Dean, P. (1996). Impaired performance of children with dyslexia on a range of cerebellar tasks. Annals of Dyslexia, 46, 259-283.

Gollwitzer, P. M. (1993). Goal achievement: The role of intentions. European Review of Social Psychology, 4, 141-185.

Gollwitzer, P. M. (1996). The volitional benefits of planning. In P. M. Gollwitzer \& J. A. Bargh (Eds.), The psychology of action: Linking cognition and motivation to behavior (pp. 287-312). New York: Guilford.

Gollwitzer, P. M. (1999). Implementation intentions: Strong effects of simple plans. American Psychologist, 54, 493-503.

Gollwitzer, P. M., \& Schaal, B. (1998). Meta-cognition in action: The importance of implementation intentions. Personality and Social Psychology Review, 2, 124-136.

Lengfelder, A., \& Gollwitzer, P. M. (2001). Reflective and reflexive action control in patients with frontal brain lesions. Neuropsychology, 15, 80-100.

Logan, G. D. (1980). Attention and automaticity in Stroop and priming tasks: Theory and data. Cognitive Psychology, 12, 523-553.

MacLeod, C. M. (1991). Half a century of research on the Stroop effect: An integrative review. Psychological Bulletin, 109, 163-203.

Mayer, J. D., \& Gaschke, Y. N. (1988). The experience and metaexperience of mood. Journal of Personality and Social Psychology, $55,102-111$.
Muraven, M., \& Baumeister, R. F. (2000). Self-regulation and depletion of limited resources. Does self-control resemble a muscle? Psychological Bulletin, 126, 247-259.

Muraven, M., Baumeister, R. F., \& Tice, D. M. (1999). Longitudinal improvement of self-regulation through practice: Building selfcontrol strength through repeated exercise. The Journal of Social Psychology, 139, 446-457.

Muraven, M., Tice, D. M., \& Baumeister, R. F. (1998). Self-control as a limited resource: Regulatory depletion patterns. Journal of Personality and Social Psychology, 74, 774-789.

Pere Ubu (Band). (1998). Modern Dance (CD Recording No. COOK CD 141). London, UK: Cooking Vinyl Records.

Sheeran, P. (2002). Intention-behavior relations: A conceptual and empirical review. In M. Hewstone \& W. Stroebe (Eds.), European review of social psychology (Vol. 12, pp. 1-36). Chichester, England: Wiley.

Sheeran, P., \& Orbell, S. (1999). Implementation intentions and repeated behavior: Augmenting the predictive validity of the theory of planned behavior. European Journal of Social Psychology, 29, 349-369.

Sheeran, P., \& Orbell, S. (2000). Using implementation intentions to increase attendance for cervical cancer screening. Health Psychology, 19, 283-289.

Smets, E. M., A, Garssen, B., Bonke, B., \& De Haes, J. C. J. M. (1995). The multi-dimensional fatigue inventory (MFI): Psychometric qualities of an instrument to assess fatigue. Journal of Psychosomatic Research, 39, 315.

Stroop, J. R. (1935). Studies of interference in serial verbal reactions. Journal of Experimental Psychology, 18, 643.

Tice, D. M., Bratlavsky, E., \& Baumeister, R. F. (2001). Emotional distress regulation takes precedence over impulse control: If you feel bad, just do it! Journal of Personality and Social Psychology, 80, 53-67. 